\title{
Visiting bijective digitized reflections and rotations using geometric algebra
}

\author{
Stéphane Breuils ${ }^{1}[0000-0002-8636-4977]$, Yukiko Kenmochi ${ }^{2}[0000-0001-9648-326 X]$, \\ and Akihiro Sugimoto ${ }^{1[0000-0001-9148-9822]}$ \\ 1 National Institute of Informatics, Tokyo, Japan \\ \{breuils, sugimoto\}@nii.ac.jp \\ 2 LIGM, Univ Gustave Eiffel, CNRS, France \\ yukiko.kenmochi@esiee.fr
}

\begin{abstract}
Geometric algebra has become popularly used in applications dealing with geometry. This framework allows us to reformulate and redefine problems involving geometric transformations in a more intuitive and general way. In this paper, we focus on 2D bijective digitized reflections and rotations. After defining the digitization through geometric algebra, we characterize the set of bijective digitized reflections in the plane. We derive new bijective digitized rotations as compositions of bijective digitized reflections since any rotation is represented as the composition of two reflections. We also compare them with those obtained through geometric transformations by computing their distributions.
\end{abstract}

\section{Introduction}

Bijectivity of digitized rotations in two and three dimensions has been studied. Its characterization was initiated by the work on two-dimensional rotations followed by a digitized operator in the square grid [8|9]. It was then shown in [12] that an arithmetic proof of the characterization is provided through Gaussian integers. Similar arithmetic characterization on the hexagonal grid was also shown using the Eisenstein integers [11]. Concerning digitized rotations in the space, using the Lipschitz quaternions [7] allows to verify the bijectivity of a given digitized rotation [10]. A bijective reflection algorithm over the plane, on the other hand, was proposed in [3] where the line of reflection is digitized.

These arithmetic approaches using algebraic numbers are intuitive and convenient for providing the proofs of the characterization/certification of the bijective digitized rotations. However, each algebraic number provides different operations and definitions, so that they cause the lack of generality and extensiblity. In contrast, geometric algebra is designed to retain generality and offer operators that are capable of computing considered geometric transformations for any geometric objects of the algebra in any dimension. This algebra was defined thanks to the work of Clifford [4] to unify and generalize Grassmann algebra and Hamilton's quaternion into a whole algebra. Geometric algebra is a framework that encompasses both quaternion algebra and complex numbers 
and extends rigid transformations of geometric objects to higher dimensions by expressing them as composition of reflections. We therefore consider that geometric algebra is a natural tool for reasoning with both digitized reflections and rotations.

In order to exploit the generality and extensibility of geometric algebra for tackling digital geometry problems, we first formulate digitization of reflections and rotations in $n$ dimensions using geometric algebra. We then focus on two dimensions and study bijectivity of digitized reflections. We then show that composition of bijective digitized reflections results in new bijective digitized rotations, allowing us to approximate any digitized rotation by bijective digitized rotations.

\section{Reflections and rotations via geometric algebra}

Geometric algebra of a vector space is an algebra over a field such that its multiplication called geometric product is defined on a space of elements, i.e., multivectors [6]. Geometric algebra is an intuitive and geometric object-oriented algebra that allows to define geometric transformations in an efficient way. Definitions and compositions of geometric transformations are given through geometric products which are invertible. Let us briefly review geometric product rules.

\subsection{Geometric product}

Given two vectors $\mathbf{m}, \mathbf{n}$, the geometric product is defined as

$$
\mathbf{m n}=\mathbf{m} \cdot \mathbf{n}+\mathbf{m} \wedge \mathbf{n},
$$

where $\mathbf{m} \cdot \mathbf{n}=\|\mathbf{m}\|\|\mathbf{n}\| \cos (\alpha)$ and $\mathbf{m} \wedge \mathbf{n}=\|\mathbf{m}\|\|\mathbf{n}\| \sin (\alpha) \mathbf{I}$ with angle $\alpha$ between $\mathbf{m}$ and $\mathbf{n}$, and $\mathbf{I}$ as the bivector basis spanned by $\mathbf{m}$ and $\mathbf{n}$. Briefly, a bivector (or 2-vector) is an element of the algebra different from a scalar and a vector such that it geometrically represents an oriented area spanned by two vectors. Here the bivector I represents the unit oriented area element of the plane spanned by the vectors $\mathbf{m}$ and $\mathbf{n}$.

Letting $d$ be the dimension of the vector space, the geometric product acts on the basis vectors $\mathbf{e}_{i}, \mathbf{e}_{j}$ and basis bivectors $\mathbf{e}_{i j}(i, j \in[1, d])$ as follows:

$$
\mathbf{e}_{i} \mathbf{e}_{j}=\left\{\begin{array}{cl}
1 & \text { if } i=j \\
-\mathbf{e}_{j i} & \text { otherwise }
\end{array} \text { and } \mathbf{e}_{i j} \mathbf{e}_{k}=\left\{\begin{array}{c}
\mathbf{e}_{i j k} \text { if } j \neq k, i \neq k \\
\mathbf{e}_{i} \text { if } j=k \\
-\mathbf{e}_{j} \text { if } i=k
\end{array} .\right.\right.
$$

If we permute $\mathbf{e}_{k}$ and $\mathbf{e}_{i j}$, the above multiplication becomes

$$
\mathbf{e}_{k} \mathbf{e}_{i j}=\left\{\begin{array}{l}
\mathbf{e}_{k i j} \text { if } j \neq k, i \neq k \\
-\mathbf{e}_{i} \text { if } j=k \\
\mathbf{e}_{j} \text { if } i=k
\end{array} .\right.
$$


Given a vector $\mathbf{x}$ defined as the weighted sum of components over the basis vectors, namely, $\mathbf{x}=\sum_{i=1, \ldots, d} u_{i} \mathbf{e}_{i}$ the norm of $\mathbf{x}$ is defined as $\|\mathbf{x}\|=\sqrt{\mathbf{x} \cdot \mathbf{x}}=$ $\sqrt{\sum_{i=1, \ldots, d} u_{i}^{2}}$. Then, by definition, the inverse of $\mathbf{x}$ is defined as $\mathbf{x}^{-1}=\frac{1}{\mathbf{x}}=$ $\frac{\mathbf{x}}{\|\mathbf{x}\|^{2}}=\frac{\mathbf{x}}{\mathbf{x} \mathbf{x}}$. The geometric product is invertible. In addition, the geometric product is associative and distributive over the addition but not commutative. The inner product results in a scalar. Namely, for given $\mathbf{e}_{i}, \mathbf{e}_{j}$

$$
\mathbf{e}_{i} \cdot \mathbf{e}_{j}= \begin{cases}1 & \text { if } i=j \\ 0 & \text { otherwise }\end{cases}
$$

\subsection{Reflections}

A reflection is the isometric mapping from $\mathbb{R}^{n}$ to itself with a hyperplane as a set of fixed (invariant) points. It is defined as follows with geometric algebra when the hyperplane goes through the origin.

Definition 1. Given a hyperplane passing through the origin, with its normal vector $\mathbf{m} \in \mathbb{R}^{d}$, denoted by $H(\mathbf{m})$, the reflection of point $\mathbf{x} \in \mathbb{R}^{n}$ with respect to $H(\mathbf{m})$ is defined as

$$
\mid \begin{aligned}
\mathcal{U}^{\mathbf{m}}: \mathbb{R}^{d} & \rightarrow \quad \mathbb{R}^{d} \\
\mathbf{x} & \mapsto-\mathbf{m} \mathbf{x m} \mathbf{m}^{-1}=-\frac{1}{\|\mathbf{m}\|^{2}} \mathbf{m} \mathbf{x m} .
\end{aligned}
$$

Reflections $\mathcal{U}^{\mathbf{m}}$ are said rational if all the components of $\mathbf{m}$ are rational. Note that any rational reflection $\mathcal{U}^{\mathbf{m}}$ can be represented by $\mathbf{m}=\sum_{i=1 \ldots d} u_{i} \mathbf{e}_{i}$ such that $u_{i} \in \mathbb{Z}$ and $\operatorname{gcd}\left(u_{1}, \cdots, u_{d}\right)=1$.

\subsection{Rotations}

Any rotation is expressed as the composition of two reflections with geometric algebra. If a first reflection w.r.t. $H(\mathbf{m})$ followed by a second reflection w.r.t. $H(\mathbf{n})$, is applied to point $\mathbf{x} \in \mathbb{R}^{d}$, we have point $\mathbf{x}^{\prime}$ such that

$$
\mathbf{x}^{\prime}=-\mathbf{n}\left(-\mathbf{m} \mathbf{x m} \mathbf{m}^{-1}\right) \mathbf{n}^{-1}=(\mathbf{n m}) \mathbf{x}(\mathbf{n m})^{-1} .
$$

In other words, $\mathbf{x}^{\prime}$ is the rotation of $\mathbf{x}$ around the intersection of $\mathbf{m}$ and $\mathbf{n}$. Indeed, assuming $\mathbf{n}$ and $\mathbf{m}$ are both normalized, we have

$$
\mathbf{x}^{\prime}=(\cos \phi+\sin \phi \mathbf{I}) \mathbf{x}(\cos \phi-\sin \phi \mathbf{I}),
$$

where $\phi$ is the angle between $\mathbf{n}$ and $\mathbf{m}$ in the rotation plane whose bivector is $\mathbf{I}$ (cf. Eq. (1)). Note that the angle of this rotation corresponds to $2 \phi$.

More generally, the algebraic entity representing the rotation of angle $\theta$ in the rotation plane whose bivector is $\mathbf{I}$ is defined as

$$
Q=\cos \frac{\theta}{2}+\sin \frac{\theta}{2} \frac{\mathbf{I}}{\|\mathbf{I}\|} \text {. }
$$




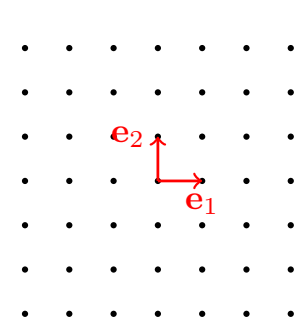

(a)

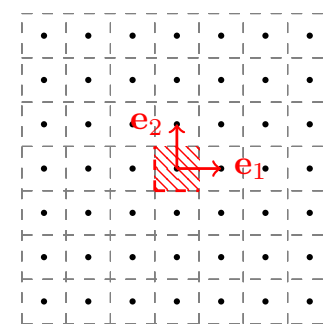

(b)

Fig. 1: (a) shows the set of points (denoted with small black point) in the digital square grid $\mathbb{Z} \mathbf{e}_{1} \oplus \mathbb{Z} \mathbf{e}_{2}$ (also denoted by $\mathbb{Z}^{2}$ ). Such points are obtained as linear combinations of the basis vectors $\mathbf{e}_{1}$ and $\mathbf{e}_{2}$ illustrated with red arrow. (b) shows digitization cells associated to the square grid using gray dashed square. The red hatched square denote the digitization cell associated with the origin or $\mathcal{C}(\mathbf{0})$; the border of $\mathcal{C}(\mathbf{0})$ is denoted with red circle.

Then, a point $\mathbf{x}$ is rotated to $\mathbf{x}^{\prime}$ as follows:

$$
\mathbf{x}^{\prime}=Q \mathbf{x} Q^{\dagger}
$$

where $Q^{+}=\cos \left(\frac{\theta}{2}\right)-\sin \frac{\theta}{2} \frac{\mathbf{I}}{\|\mathbf{I}\|}$. Note that $\mathbf{I}=\mathbf{e}_{12}$ in the $2 \mathrm{D}$ case.

\section{Digitized reflections}

In order to define digitized reflection, we need to define the grid to digitize points.

\subsection{Cubic grids}

In a similar way as the state-of-the-art, we denote the set of vectors of geometric algebra in the space with real coordinates as

$$
\mathbb{R}^{d}=\left\{\mathbf{x}=\sum_{i=1, \ldots, d} u_{i} \mathbf{e}_{i} \mid u_{i} \in \mathbb{R}\right\}
$$

Those with integer coordinates are called the cubic grid or the integer lattice in $\mathbf{R}^{d}$, defined as

$$
\mathbb{Z}^{d}=\left\{\mathbf{x}=\sum_{i=1, \ldots, d} a_{i} \mathbf{e}_{i} \mid a_{i} \in \mathbb{Z}\right\}
$$

This cubic grid is also written as $\mathbb{Z}^{d}=\oplus_{i=1, \ldots, d} \mathbb{Z} \mathbf{e}_{i}$. An illustration of such a cubic grid in the plane is given in Figure $1 \mathrm{a}$

By extension, any square grid generated by two orthogonal vectors, $u \mathbf{e}_{1}+$ $v \mathbf{e}_{2}$ and $-v \mathbf{e}_{1}+u \mathbf{e}_{2}$, in the plane is defined as:

$$
\mathbb{Z}\left(u \mathbf{e}_{1}+v \mathbf{e}_{2}\right) \oplus \mathbb{Z}\left(-v \mathbf{e}_{1}+u \mathbf{e}_{2}\right) .
$$




\subsection{Digitization of reflections}

A digitized reflection is a reflection followed by a digitization. Let us consider reflections of points in $\mathbb{Z}^{d}$.

As $\mathcal{U}^{\mathbf{m}}\left(\mathbb{Z}^{d}\right) \nsubseteq \mathbb{Z}^{d}$ in general, we need to define the digitization operator again on the cubic grid in order to obtain digitized reflection points. To this end, we first define digitization cells of the cubic grid $\mathbb{Z}^{d}$.

Definition 2. For any $\kappa \in \mathbb{Z}^{d}$, we define the digitization cell of $\kappa$ as

$$
\begin{aligned}
\mathcal{C}(\kappa):=\left\{\mathbf{x} \in \mathbb{R}^{d} \mid \forall i \in[1, d]\|\mathbf{x}-\kappa\|\right. & \leq\left\|\mathbf{x}-\kappa+\mathbf{e}_{i}\right\| \\
\text { and }\|\mathbf{x}-\kappa\| & \left.<\left\|\mathbf{x}-\kappa-\mathbf{e}_{i}\right\|\right\} .
\end{aligned}
$$

This can be rewritten as:

$$
\mathcal{C}(\kappa):=\left\{\mathbf{x}=\sum_{i=1, \ldots, d} x_{i} \mathbf{e}_{i} \in \mathbb{R}^{d} \mid \forall i \in[1, d] a_{i}-\frac{1}{2} \leq x_{i}<a_{i}+\frac{1}{2}\right\},
$$

where $\kappa=\sum_{i=1, \ldots, d} a_{i} \mathbf{e}_{i}$. An example of the set of digitization cells obtained from the square grid of Figure $1 \mathrm{a}$ is shown in Figure $1 \mathrm{~b}$.

We also define the digitization cell associated to a transformation such as reflection, rotation, and scaling.

Definition 3. Given a transformation $\mathcal{T}$ such that any basis vector $\mathbf{e}_{i}$ is transformed to $\mathcal{T} \mathbf{e}_{i} \mathcal{T}^{\dagger}$, the digitization cell of $\kappa \in \mathbb{Z}^{d}$ transformed by $\mathcal{T}$ is defined as

$$
\begin{aligned}
\mathcal{C}_{\mathcal{T}}(\kappa):=\left\{\mathbf{x} \in \mathbb{R}^{d} \mid \forall i \in[1, d]\|\mathbf{x}-\kappa\|\right. & \leq\left\|\mathbf{x}-\kappa+\mathcal{T} \mathbf{e}_{i} \mathcal{T}^{\dagger}\right\| \\
\text { and }\|\mathbf{x}-\kappa\| & \left.<\left\|\mathbf{x}-\kappa-\mathcal{T} \mathbf{e}_{i} \mathcal{T}^{\dagger}\right\|\right\} .
\end{aligned}
$$

Note that Definition 3 covers:

- a reflected digital cell, if $\mathcal{T}=\sum_{i=1, \ldots, d} u_{i} \mathbf{e}_{i}$ with $\sum_{i=1, \ldots, d} u_{i}^{2}=1$;

- a rotated digital cell, if $\mathcal{T}=u+v \mathbf{I}$ with $u^{2}+v^{2}=1$;

- a scaled digital cell, if $\mathcal{T}=u$ with $u \in \mathbb{R}$ and the digital cell is scaled by a factor $u^{2}$.

We also note that the non-transformed digitization cell centered in $\kappa$ is identical with the digitization cell defined in Definition $2 \mathcal{C}_{1}(\kappa)=\mathcal{C}(\kappa)$. This comes simply from the fact that the multiplication of the scalar 1 and the basis vector $\mathbf{e}_{i}$ is $1 \mathbf{e}_{i}=1 \mathbf{e}_{i}=\mathbf{e}_{i}$.

Similarly to [11], we define the digitization operator as follows:

Definition 4. The digitization operator on a cubic grid is defined as

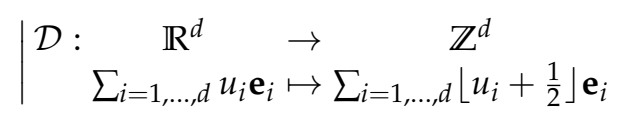

where $\lfloor u\rfloor(u \in \mathbb{R})$ denotes the greatest integer not greater than $u$. 
Now we define the digitized reflection as the composition of the reflection and the digitization.

Definition 5. Given a hyperplane $H(\mathbf{m})$, a digitized reflection with respect to $H(\mathbf{m})$ is defined as

$$
\begin{aligned}
\mathcal{R}^{\mathbf{m}}: \mathbb{Z}^{d} & \rightarrow \mathbb{Z}^{d} \\
\mathbf{x} & \mapsto \mathcal{D} \circ \mathcal{U}^{\mathbf{m}}(\mathbf{x})
\end{aligned}
$$

Hereafter we focus on the case of $d=2$.

\section{Bijective digitized reflections}

In order to describe the bijectivity of digitized reflections $\mathcal{R}^{\mathrm{m}}$ in the plane, we need to explore the structure of the square grid after reflection $\mathcal{U}^{\mathrm{m}}$. We start by the reflection of the basis vectors. Let us denote the reflection of $\mathbf{e}_{1}$ and $\mathbf{e}_{2}$ with respect to $H(\mathbf{m})$ by $\boldsymbol{\phi}$ and $\boldsymbol{\psi}$, respectively. Applying Definition 11 to $\mathbf{e}_{1}$ and $\mathbf{e}_{2}$ results in

$$
\begin{aligned}
& \boldsymbol{\phi}=\mathcal{U}^{\mathbf{m}}\left(\mathbf{e}_{1}\right)=-\mathbf{m} \mathbf{e}_{1} \mathbf{m}^{-1}=\frac{v^{2}-u^{2}}{u^{2}+v^{2}} \mathbf{e}_{1}+\frac{2 u v}{u^{2}+v^{2}} \mathbf{e}_{2} \\
& \boldsymbol{\psi}=\mathcal{U}^{\mathbf{m}}\left(\mathbf{e}_{2}\right)=-\mathbf{m e}_{2} \mathbf{m}^{-1}=\frac{2 u v}{u^{2}+v^{2}} \mathbf{e}_{1}+\frac{u^{2}-v^{2}}{u^{2}+v^{2}} \mathbf{e}_{2}
\end{aligned}
$$

Reflection of any point $\mathbf{y} \in \mathbb{Z}^{2}$ is expressed as a linear combination of the reflected unit vectors $\phi$ and $\boldsymbol{\psi}$. Namely, the reflected points of $\mathbb{Z} \mathbf{e}_{1}+\mathbb{Z} \mathbf{e}_{2}$ are the points of the grid $\mathbb{Z} \boldsymbol{\phi}+\mathbb{Z} \boldsymbol{\psi}$. An example of the transformed grid is shown in Figure2 2

\subsection{Set of remainders}

In a similar way as in [12], let us first consider the set of remainders to give the definition of bijective reflections.

Definition 6. Given a reflection $\mathcal{U}^{\mathbf{m}}$, the set of remainders $\mathcal{S}^{\mathbf{m}}$ is defined as

$$
\begin{aligned}
\mathcal{S}^{\mathbf{m}}: \mathbb{Z}^{2} \times \mathbb{Z}^{2} & \rightarrow \\
(\mathbf{x}, \mathbf{y}) & \mapsto \mathcal{U}^{\mathbf{m}}(\mathbf{x})-\mathbf{y}
\end{aligned}
$$

Definition 7. A digitized reflection $\mathcal{R}^{\mathrm{m}}=\mathcal{D} \circ \mathcal{U}^{\mathbf{m}}$ is bijective if and only if

$$
\forall \mathbf{y} \in \mathbb{Z}^{2}, \exists ! \mathbf{x} \in \mathbb{Z}^{2}, \mathcal{S}^{\mathbf{m}}(\mathbf{x}, \mathbf{y}) \in \mathcal{C}_{1}(\mathbf{0}),
$$

where $\mathbf{0}$ corresponds to the null vector. 


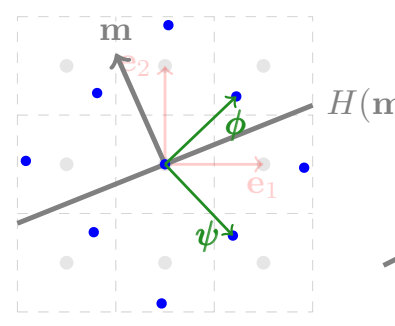

(a)

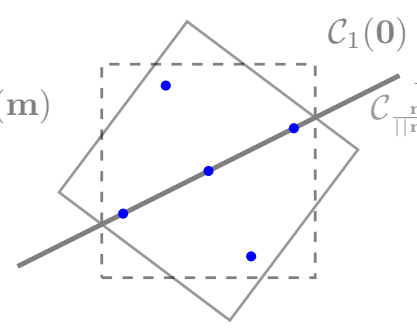

(b)

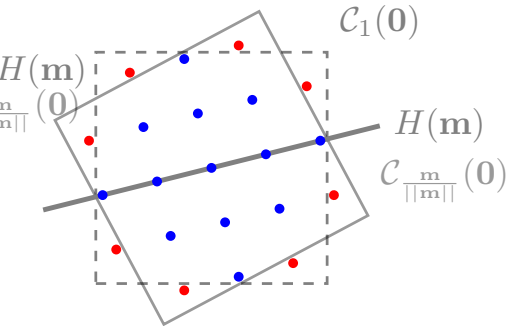

(c)

Fig. 2: (a) Discrete square grid $\mathbb{Z} \mathbf{e}_{1} \oplus \mathbb{Z} \mathbf{e}_{2}$ illustrated by gray dots, and their associated digitization cells illustrated by gray dashed squares, using the geometric algebra implementation ganja.js [5]. The reflected points with respect to the line $H(\mathbf{m})$, i.e., $\mathbb{Z} \boldsymbol{\phi}+\mathbb{Z} \boldsymbol{\psi}$, illustrated by blue dots. (b) and (c) Set of remainders illustrated by blue (and red) dots; in (b) the set of remainders satisfies the bijectivity condition while (c) does not (see the red dots for non-bijective points).

Note that this definition can be divided into two parts like [12]:

$$
\left\{\begin{array}{l}
\forall \mathbf{y} \in \mathbb{Z}^{2}, \exists \mathbf{x} \in \mathbb{Z}^{2}, \mathcal{S}^{\mathbf{m}}(\mathbf{x}, \mathbf{y}) \in \mathcal{C}_{1}(\mathbf{0}) \\
\forall \mathbf{x} \in \mathbb{Z}^{2}, \exists \mathbf{y} \in \mathbb{Z}^{2}, \mathcal{S}^{\mathbf{m}}(\mathbf{x}, \mathbf{y}) \in \mathcal{C}_{\|\mathbf{m}\|}(\mathbf{0})
\end{array}\right.
$$

provided $\mathcal{S}^{\mathbf{m}}\left(\mathbb{Z}^{2}, \mathbb{Z}^{2}\right) \cap \mathcal{C}_{1}(\mathbf{0})=\mathcal{S}^{\mathbf{m}}\left(\mathbb{Z}^{2}, \mathbb{Z}^{2}\right) \cap \mathcal{C}_{\frac{\mathbf{m}}{\|\mathbf{m}\|}}(\mathbf{0})$, that is to say:

$$
\mathcal{I}=\mathcal{S}^{\mathbf{m}}\left(\mathbb{Z}^{2}, \mathbb{Z}^{2}\right) \cap\left(\mathcal{C}_{1}(\mathbf{0}) \cup \mathcal{C}_{\|\mathbf{m}\|}(\mathbf{0})\right) \backslash\left(\mathcal{C}_{1}(\mathbf{0}) \cap \mathcal{C}_{\|\mathbf{m}\|}(\mathbf{0})\right)=\varnothing .
$$

As an illustration, Fig. $2 \mathrm{~b}$ verifies the above condition whereas Fig. $2 \mathrm{c}$ does not.

\subsection{Non-rational reflection}

We first show that non-rational digitized reflections are not bijective. To achieve this, we study the structure of the set of remainders $\mathcal{S}^{\mathbf{m}}\left(\mathbb{Z}^{2}, \mathbb{Z}^{2}\right)$ with respect to the parameters of the digitized reflection.

$\mathcal{G}$ denotes the set composed of the lattice $\mathbb{Z} \mathbf{e}_{1} \oplus \mathbb{Z} \mathbf{e}_{2}$ and $\mathbb{Z} \boldsymbol{\phi} \oplus \mathbb{Z} \boldsymbol{\psi}$ :

$$
\mathcal{G}=\mathbb{Z} \mathbf{e}_{1} \oplus \mathbb{Z} \mathbf{e}_{2} \oplus \mathbb{Z} \boldsymbol{\phi} \oplus \mathbb{Z} \boldsymbol{\psi}
$$

Proposition 1. If the digitized reflection $\mathcal{U}^{\mathrm{m}}$ is non-rational, the set $\mathcal{G}$ is dense and infinite.

Non-rational digitized reflection means the reflected components computed in Eq.(10) and Eq.(11), are not integers (non-Pythagorean primitive triples). From [9], the set obtained with non-Pythagorean is dense and infinite. In such a 
case the digitized reflection is not bijective since the two vectors $\mathbf{a}, \mathbf{b} \in \mathcal{S}^{\mathbf{m}}\left(\mathbb{Z}^{2}, \mathbb{Z}^{2}\right) \cap$ $\mathcal{C}_{1}(\mathbf{0})$ exist such that $\mathcal{S}^{\mathbf{m}}(\mathbf{x}, \mathbf{y})=\mathbf{a}$ and $\mathcal{S}^{\mathbf{m}}\left(\mathbf{x}+\mathbf{e}_{1}, \mathbf{y}\right)=\mathbf{b}$ (consequence of the Bolzano-Weirstrass theorem). This violates the bijectivity condition because both $\mathcal{R}^{\mathbf{m}}(\mathbf{x}) \in \mathcal{C}_{1}(\mathbf{y})$ and $\mathcal{R}^{\mathbf{m}}\left(\mathbf{x}+\mathbf{e}_{1}\right) \in \mathcal{C}_{1}(\mathbf{y})$.

\subsection{Bijectivity of digitized reflections}

In order to characterize normal vectors leading to bijective digitized reflections, we use the bijectivity condition of digitized rotations. Let us first recall the bijectivity condition defined in [9] with primitive Pythagorean triples. A digitized rotation whose rotation angle is $\theta$ is bijective if and only if

$$
\{\cos (\theta), \sin (\theta)\}=\left\{\frac{2 k+1}{2 k^{2}+2 k+1}, \frac{2 k(k+1)}{2 k^{2}+2 k+1}\right\}, k \in \mathbb{N} .
$$

Using the half angle formula and assuming $\theta \in\left[0, \frac{\pi}{4}\right]$, we have

$$
\begin{aligned}
\cos \left(\frac{\theta}{2}\right) & =\sqrt{\frac{1}{2}+\frac{2 k+1}{2\left(2 k^{2}+2 k+1\right)}}, & \sin \left(\frac{\theta}{2}\right) & =\sqrt{\frac{1}{2}-\frac{2 k+1}{2\left(2 k^{2}+2 k+1\right)}} . \\
& =\frac{(k+1)}{\sqrt{2 k^{2}+2 k+1}} & & =\frac{k}{\sqrt{2 k^{2}+2 k+1}}
\end{aligned}
$$

With Eq. (7), this can be rewritten using the digitized rotation given by the entity $\mathbf{Q}$ as

$$
\mathbf{Q}=(k+1)+k \mathbf{e}_{12}, \quad k \in \mathbb{N} .
$$

Note that the rotation operator does not change by any scale.

Conversely, from Eq. (17) we have

$$
\begin{aligned}
\mathbf{Q x} \mathbf{Q}^{-1} & =\left(((k+1) x+k y) \mathbf{e}_{1}+((k+1) y-k x) \mathbf{e}_{2}\right)\left(\frac{k+1-k \mathbf{e}_{12}}{2 k^{2}+2 k+1}\right) \\
& =\frac{1}{2 k^{2}+2 k+1}((2 k+1) x-2 k(k+1) y) \mathbf{e}_{1}+((2 k+1) y+2 k(k+1) x) \mathbf{e}_{2}
\end{aligned}
$$

which leads to Eq. (16). This indicates that Eq. 17) and Eq. (16) are equivalent with each other. Eq. (17) is thus the bijectivity condition of digitized rotations with geometric algebra. We remark that Gaussian integers defined in [12] gives us similar argument.

Proposition 2. Given a rational reflection line $H(\widetilde{\mathbf{m}})$ such that

$$
\widetilde{\mathbf{m}}=-k \mathbf{e}_{1}+(k+s) \mathbf{e}_{2}, \quad k \in \mathbb{N}, s \in \mathbb{N},
$$

the rational digitized reflection $\mathcal{R}^{\widetilde{\mathrm{m}}}$ is bijective if and only if $s=1$.

Proof. The idea is simply to express the set of remainders of digital reflections $\mathcal{S}^{\widetilde{\mathbf{m}}}$ by the set of remainders of digitized rotations $\mathcal{S}^{Q}$ where $Q$ is a digitized rotation entity. This is performed through the fact that a composition of any digitized reflection and the digitized reflection with respect to $H\left(\mathbf{e}_{2}\right.$ does not induce any change in the set of remainders, namely

$$
\mathcal{S}^{\mathbf{e}_{2} \mathbf{m}}\left(\mathbb{Z}^{2}, \mathbb{Z}^{2}\right)=\mathcal{S}^{\mathbf{m}}\left(\mathbb{Z}^{2}, \mathbb{Z}^{2}\right)
$$




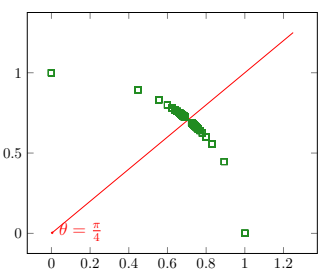

(a)

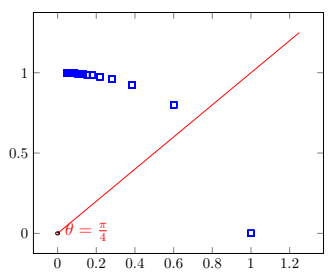

(b)

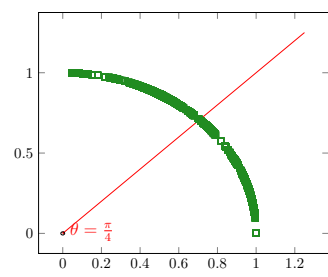

(c)

Fig. 3: Distribution of (reflection and rotation) angles within $\left[0, \frac{\pi}{2}\right]$ that make the digitized transformations bijective: (a) digitized reflections, (b) digitized rotations, (c) digitized rotation approximations through pairs of bijective digitized reflections. The same integer parameter $k_{\max }=20$ was used for the three figures. Note that the distribution of angles in $\left[\frac{\pi}{4}, \frac{\pi}{2}\right]$ is obtained by the reflection of that in $\left[0, \frac{\pi}{4}\right]$ with respect to the line $x=y$.

Besides,

$$
\mathcal{C}_{\widetilde{\tilde{m}}}(\mathbf{m})=\mathcal{C}_{\mathbf{e}_{2} \tilde{\mathbf{m}} \|}(\mathbf{m})
$$

Algebraically $\mathbf{e}_{2} \mathbf{m}=\mathbf{e}_{2}\left(-k \mathbf{e}_{1}+(k+s) \mathbf{e}_{2}\right)=(k+s)+k \mathbf{e}_{12}$. The resulting entity is homogeneous to a rotation. From Eq. (17), this entity is bijective if and only if $s=1$.

\subsection{Finding the closest bijective digitized reflections}

Let us consider the set of all bijective digitized reflections such that the reflection lines have slant angles $\theta \in\left[0, \frac{\pi}{4}[\right.$ :

$$
\mathbf{B}=\left\{\mathcal{U}^{\widetilde{\mathbf{m}}} \mid \widetilde{\mathbf{m}}=-k \mathbf{e}_{1}+(k+1) \mathbf{e}_{2}, k \in \mathbb{N}\right\} .
$$

We show in Fig. 3a that the slant angles of such reflection lines defined by $\widetilde{\mathbf{m}}$ are sparse around $\theta=0$ while dense around $\theta=\frac{\pi}{4}$. We remark that in practice, we have to limit the maximum value $k_{\max }$ of $k$ because of the image size. Hereafter, instead of $\mathbf{B}$, we use $\mathbf{B}_{k_{\max }}$ with the condition $k \leq k_{\max }$.

The sparsity of $\mathbf{B}_{k_{\max }}$ motivates us to approximate a given reflection $\mathcal{R}^{\mathbf{m}}$ with the closest digitized reflection $\mathcal{R}^{\widetilde{\mathbf{m}}}$ such that $\mathcal{U}^{\widetilde{\mathbf{m}}} \in \mathbf{B}_{k_{\max }}$. More precisely, given $\mathbf{m}$ with slant angle $\theta$ of its reflection line, we seek for $\widetilde{\mathbf{m}}$ with reflection line having the slant angle $\widetilde{\theta}$ that minimizes the absolute difference between the angles:

$$
\underset{\mathcal{U}^{\widetilde{\mathbf{m}}} \in \mathbf{B}_{k \max }}{\arg \min }|\widetilde{\theta}-\theta| .
$$




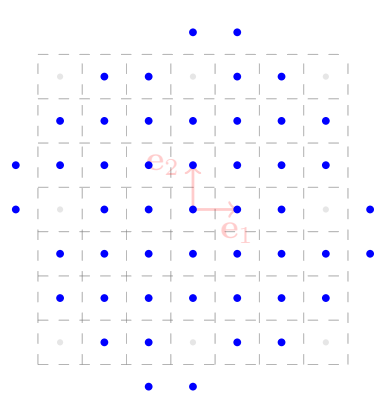

(a)

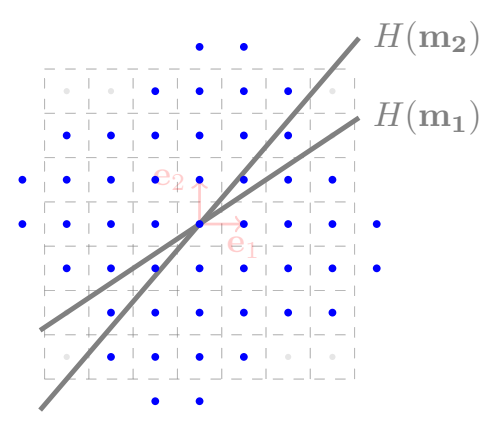

(b)

Fig. 4: (a) digitized rotation with $\theta=\frac{\pi}{6}$ which yields holes and double points. (b) same digitized rotation approximation with composition of two bijective reflections.

Since tan monotonically increases in $\left[-\frac{\pi}{4}, \frac{\pi}{4}\right]$, we can consider $|\tan (\widetilde{\theta}-\theta)|$ instead of $|\widetilde{\theta}-\theta|$. This minimization is thus equivalent with

$$
\underset{\mathcal{U}^{\tilde{\mathbf{m}} \in \mathbf{B}_{k_{\max }}}}{\arg \min }\left|\frac{\tan (\widetilde{\theta})-\tan (\theta)}{1+\tan (\widetilde{\theta}) \tan (\theta)}\right|=\underset{\widetilde{k} \in \mathbb{N}}{\arg \min }\left|\frac{\widetilde{k} x-(\widetilde{k}+1) y}{(\widetilde{k}+1) x+\widetilde{k} y}\right|,
$$

where $(x, y)$ are the components of $\mathbf{m}$, i.e., $\tan \theta=y / x(x>y)$. Assuming $\widetilde{k} \in \mathbb{Q}_{+}$, we find that $\widetilde{k}=\frac{y}{x-y}$ achieves the minimum of the objective function by making its numerator 0 because the denominator is always positive. As the function $f(k)=(k x-(k+1) y) /((k+1) x+k y)$ is increasing for all $k \geq 0$, $f(k) \leq 0$ when $0 \leq k \leq y /(x-y)$ and $f(k)>0$ otherwise, we can find $\widetilde{k} \in \mathbb{N}$ such as

$$
\widetilde{k}=\underset{\widetilde{k} \in\left\{\left\lfloor\frac{y}{x-y}\right\rfloor,\left[\frac{y}{x-y}\right\rceil\right\}}{\arg \min }\left|\frac{\widetilde{k} x-(\widetilde{k}+1) y}{(\widetilde{k}+1) x+\widetilde{k} y}\right| .
$$

Note that we consider the case where $x>y$. If $x=y$, we have $\widetilde{\mathbf{m}}=-\mathbf{e}_{1}+\mathbf{e}_{2}$.

\section{Bijective digitized rotations via bijective digitized reflections}

From the set of bijective digitized reflections, we can obtain the set of bijective digitized rotations since any rotation can be expressed as the composition of two reflections. We see such rotation angles are distributed sparsely as shown in Fig. $3 \mathrm{~b}$. thus most digitized rotations are likely to be non-bijective and yield holes and/or double points (see Fig. 4a). 


\subsection{Composition of bijective digitized reflections without error}

As any rotation is composed of a pair of reflections, it is easy to see that if the first digitized reflection $\mathcal{R}^{\mathrm{m}}=\mathcal{D} \circ \mathcal{U}^{\mathrm{m}}$ induces no digitization error and the second digitized reflection $\mathcal{R}^{\mathbf{n}}$ is bijective, then $\mathcal{R}^{\mathrm{n}} \circ \mathcal{R}^{\mathrm{m}}$ is a bijective digitized rotation. Such cases occur when $\mathbf{m}=\mathbf{e}_{1}, \mathbf{e}_{2}$ or $\mathbf{e}_{1}+\mathbf{e}_{2}$. However, it is also easy to see that such composed bijective digitized rotations have the same rotation angle distribution as that of direct (non-composed) bijective digitized rotations, which is illustrated in Figure $3 \mathrm{~b}$. This leads us to investigate the composition of two general bijective digitized reflections.

\subsection{Approximating digitized rotations with bijective digitized reflections}

Any composition of bijective digitized reflections is also bijective, that is $\mathcal{R}^{\mathbf{n}}$ 。 $\mathcal{R}^{\mathrm{m}}$ is bijective if $\mathcal{R}^{\mathrm{n}}$ and $\mathcal{R}^{\mathrm{m}}$ are both bijective. Based on this fact, given a rotation angle $\theta$, the aim here is to find the best approximated rotation composed of a pair of bijective digitized reflections.

The idea of our algorithm for this is simple (see Algorithm 1). Given a maximum possible integer $k_{\max }$ that defines the set of bijective digitized reflections $\mathbf{B}_{k_{\max }}$ (see Section 4.4), we first loop over all possible bijective digitized reflections $\mathcal{U}^{\mathbf{m}_{1}} \in \mathbf{B}_{k_{\max }}$. For each $\mathcal{U}^{\mathbf{m}_{1}} \in \mathbf{B}_{k_{\max }}$, the second bijective digitized reflection $\mathcal{U}^{\mathbf{m}_{2}} \in \mathbf{B}_{k_{\max }}$ is then selected such that $\arccos \mathbf{m}_{1} \cdot \mathbf{m}_{2}$ is closest to $\frac{\theta}{2}$. For that, we use the approximation method proposed in Section 4.4 We remark that since the computations in the loop run in constant time, the overall complexity of Algorithm 1 is linear with respect to $\operatorname{card}\left(\mathbf{B}_{k_{\max }}\right)$.

The proposed algorithm was implemented in $\mathrm{C}++$ and also with the library DGtal [1] for the digital geometry part. The code is available online $3^{3}$ Figure 5 shows some results on a single image with different rotation angles.
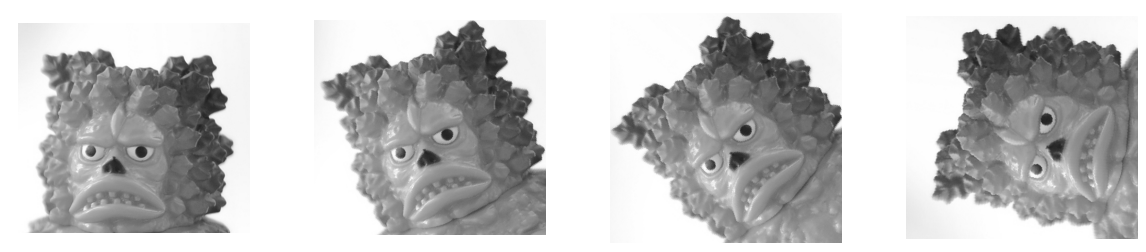

Fig. 5: Original image and its digitized rotations by bijective digitized reflections for angles $\frac{\pi}{8}, \frac{\pi}{4}, \frac{7 \pi}{16}$ from left to right.

\subsection{Distributions of bijective digitized reflections and rotations}

With a similar idea to Algorithm 1 , we can compute from $\mathbf{B}_{k_{\max }}$, all the rotation angles of such bijective approximations of digitized rotations. The distribution

\footnotetext{
3 https://github.com/sbreuils/GADigitizedTransformations.git
} 


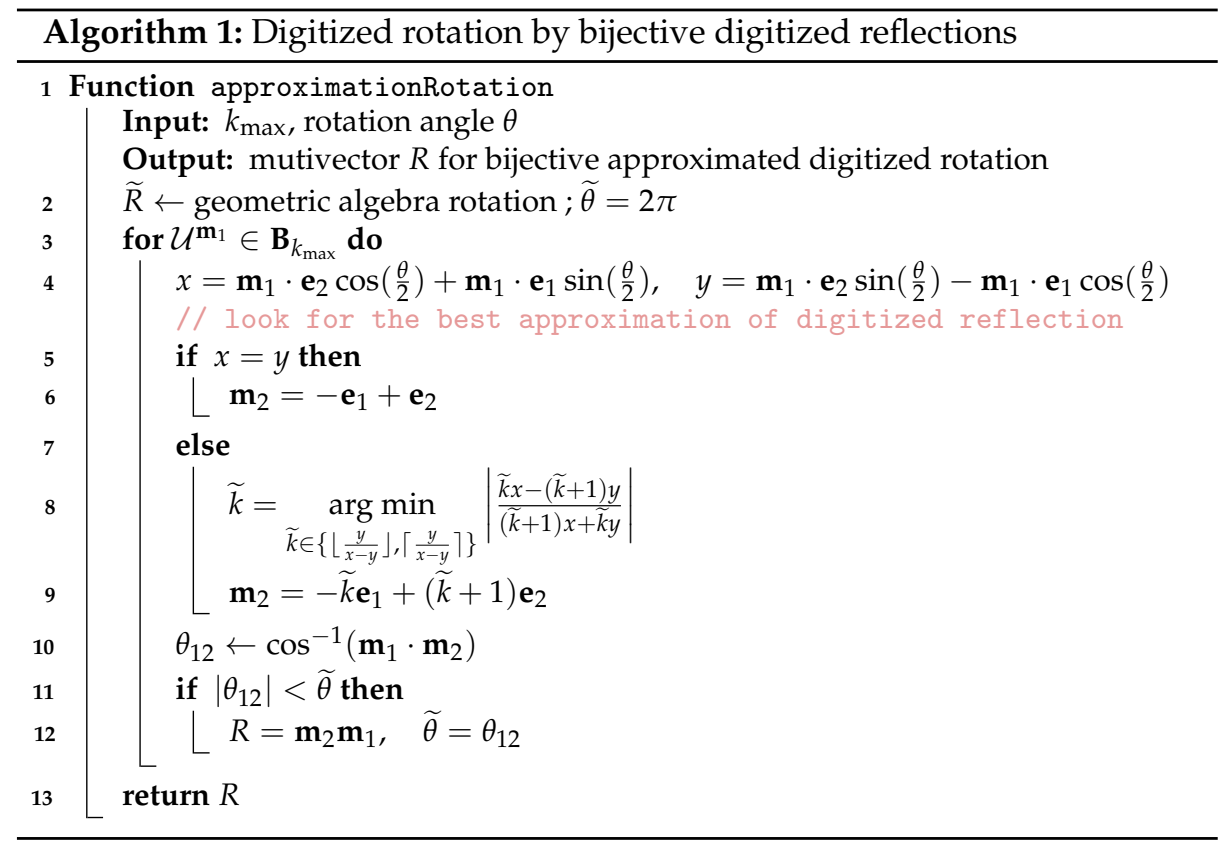

of such angles is shown in Fig. $3 \mathrm{c}$; it is even less sparse compared to those of Fig. $3 a$ and $3 b$.

In order to compare the angle distribution between Fig. $3 a$ and $3 b$, we define the angle sparsity as the maximum angle between two successive bijective digitized transformations. The angle sparsity for bijective digitized reflections is $0.46 \mathrm{rad}$ whereas it is $0.93 \mathrm{rad}$ for bijective digitized rotations. Note that they do not depend on the value of $k_{\max }$.

We can also evaluate the angle fineness (denoted by $\Delta \theta_{\min }$ ) in terms of the minimum angle between two successive bijective digitized transformations. We have from a given $k_{\max }(>1), \Delta \theta_{\min }=\arctan \left(\frac{1}{2 k_{\max }^{2}}\right)$ for bijective digitized reflections while $\Delta \theta_{\min }=\arctan \left(\frac{4 k_{\max }^{2}}{4 k_{\max }^{4}-1}\right)$ for bijective digitized rotations. Note that if $k_{\max }=1, \Delta \theta_{\min }=\arctan \left(\frac{1}{2 k_{\max }+1}\right)=\arctan \left(\frac{1}{3}\right) \approx 0.32$ rad for bijective digitized reflections while it is $\Delta \theta_{\min }=\arccos \left(\frac{2 k_{\max }\left(k_{\max }+1\right)}{2 k_{\max }^{2}+2 k_{\max }+1}\right)=\arccos \left(\frac{4}{5}\right) \approx$ $0.64 \mathrm{rad}$ for bijective digitized rotations.

We easily check that $\forall k_{\max } \in \mathbb{N}^{*}, \Delta \theta_{\min }$ of the bijective digitized reflection is lower than that of bijective digitized rotation. For example, with $k_{\max }=20$ in Fig. 3. $\Delta \theta_{\text {min }}$ of bijective digitized reflections is $1.25 \times 10^{-3}$ rad whereas that of bijective digitized rotations is $2.5 \times 10^{-3} \mathrm{rad}$. 


\section{Conclusion}

We visited reflections, rotations, and their digitization using geometric algebra. The geometric algebra framework allows us to characterize the bijective digitized reflections. We first showed that compositions of bijective digitized reflections result in new bijective digitized rotations using geometric algebra. We then demonstrated that any digitized rotation is approximated by one of these new bijective digitized rotations.

There are other approximation methods that preserve bijectivity for rotations or reflections on $\mathbb{Z}^{2}$, such as quasi-shear rotations [2] and digital bijective reflections [3]. Naturally, a comparative study of our approach with them is expected as a perspective of this article. We are also interested in adapting the presented algorithm to the case where the number of considered points of $\mathbb{Z}^{2}$ is finite; there would be more bijective digitized reflections. Finally, an extension of the concept to higher dimensions is also our interest.

\section{References}

1. DGtal: Digital geometry tools and algorithms library, https://dgtal.org/

2. Andres, E.: The Quasi-Shear rotation. In: Miguet, S., Montanvert, A., Ubéda, S. (eds.) Discrete Geometry for Computer Imagery. pp. 307-314. Springer Berlin Heidelberg, Berlin, Heidelberg (1996)

3. Andres, E., Dutt, M., Biswas, A., Largeteau-Skapin, G., Zrour, R.: Digital TwoDimensional Bijective Reflection and Associated Rotation. In: Discrete Geometry for Computer Imagery. pp. 3-14. Springer International Publishing (2019)

4. Clifford, W.K.: Applications of Grassmann's extensive algebra. American Journal of Mathematics 1(4), 350-358 (1878)

5. De Keninck, S.: ganja.js (2020). https://doi.org/10.5281/ZENODO.3635774. https : //zenodo.org/record/3635774

6. Dorst, L., Fontijne, D., Mann, S.: Geometric Algebra for Computer Science, An Object-Oriented Approach to Geometry. Morgan Kaufmann (2007)

7. Hamilton, W.R.: On quaternions; or on a new system of imaginaries in algebra. Philosophical magazine 25(3), 489-495 (1844)

8. Jacob, M.A., Andres, E.: On discrete rotations. In: 5th Int. Workshop on Discrete Geometry for Computer Imagery, Clermont-Ferrand (France). pp. 161-174. Université de Clermont-Ferrand I (September 1995)

9. Nouvel, B., Rémila, E.: Characterization of Bijective Discretized Rotations. In: International Workshop on Combinatorial Image Analysis. pp. 248-259. Springer (2004)

10. Pluta, K., Romon, P., Kenmochi, Y., Passat, N.: Bijectivity Certification of 3D Digitized Rotations. In: Computational Topology in Image Context. pp. 30-41. Springer International Publishing (2016)

11. Pluta, K., Roussillon, T., Cœurjolly, D., Romon, P., Kenmochi, Y., Ostromoukhov, V.: Characterization of bijective digitized rotations on the hexagonal grid. Journal of Mathematical Imaging and Vision 60(5), 707-716 (2018)

12. Roussillon, T., Coeurjolly, D.: Characterization of bijective discretized rotations by Gaussian integers. Research report, LIRIS UMR CNRS 5205 (2016) 